\title{
É HORA DE INVESTIRMOS EM INTERVENÇÕES COMPORTAMENTAIS COMPUTADORIZADAS NO BRASIL? ${ }^{1}$
}

\author{
Henrique Pinto Gomide ${ }^{2}$ \\ Leonardo Fernandes Martins \\ Telmo Mota Ronzani \\ Universidade Federal de Juiz de Fora, Juiz de Fora-MG, Brasil.
}

\begin{abstract}
RESUMO. Intervenções comportamentais computadorizadas apresentam evidências de eficácia para diversas condições de saúde, como transtornos de ansiedade ou de humor e abuso do uso de álcool e tabagismo. Embora apresentem efeitos relativamente pequenos, elas podem atingir um grande número de usuários simultaneamente e funcionar de forma ininterrupta. Os objetivos do presente estudo foram: 1) analisar criticamente as evidências de eficácia presentes na literatura sobre intervenções comportamentais computadorizadas; 2) avaliar o potencial de utilização no país; 3) descrever a regulamentação existente; e 4) apresentar ferramentas para desenvolvimento destas intervenções através de uma revisão narrativa da literatura. As intervenções comportamentais computadorizadas se configuram como uma potencial ferramenta para a saúde pública, devido ao crescimento do uso de computadores e internet no Brasil e ao baixo custo de seu desenvolvimento.
\end{abstract}

Palavras-chave: Aplicações do computador; modificação do comportamento; internet.

\section{IS IT TIME TO INVEST ON COMPUTERIZED BEHAVIORAL INTERVENTIONS IN BRAZIL?}

\begin{abstract}
Studies have shown that computer-based interventions are effective treatment modality for many health conditions such as anxiety disorders, depression, alcohol abuse, and nicotine dependence. Although these interventions have shown small effect sizes, they could reach a large number of people. This study aims to analyze critically the efficacy evidence of computer-based interventions in scientific literature, evaluate their potential utilization in Brazil, describe the current regulation, and introduce some development tools. To reach these aims, a narrative review of literature was performed. The computer-based interventions are configured as a potential tool for public health due to the growing use of computers and Internet in Brazil and the low cost of development.
\end{abstract}

Keywords: Computer applications; behavior modification; internet.

\section{ES HORA DE INVERTIR EN LAS INTERVENCIONES CONDUCTUALES COMPUTARIZADAS EN BRASIL?}

RESUMEN. Intervenciones conductuales informatizadas tienen evidencia de efectividad para varias condiciones de salud tales como trastornos de ansiedad, del estado de humor, el abuso del alcohol y el tabaco. A pesar de que muestran tamaños de efecto relativamente pequeñas, pueden llegar a un gran número de usuarios al mismo tiempo sin interrupción. Los objetivos de este estudio fueron: analizar críticamente las evidencias de la efectividad en la literatura presente sobre el tema, evaluar el potencial de uso en el país, describir la normativa vigente y proporcionar herramientas para el desarrollo de estas intervenciones, a través de una revisión de la literatura. Las intervenciones conductuales computarizadas se configuran como una herramienta potencial para la salud pública debido al creciente uso de computadores e internet en Brasil y el bajo costo de desarrollo.

Palabras-clave: Aplicación del computador; internet; modificación del comportamiento.

\footnotetext{
${ }^{1}$ Apoio e financiamento: Fapemig, CNPq, CAPES.

${ }^{2}$ Endereço para correspondência: Instituto de Ciências Humanas B-3-37 - Universidade Federal de Juiz de Fora - Rua José Lourenço Kelmer - s/n - Campus Universitário - São Pedro. CEP: 36036-900. Juiz de Fora-MG, Brasil. E-mail: henriquepgomide@gmail.com
} 
Transtornos mentais como ansiedade, depressão, doenças relacionadas ao uso de álcool e psicoses estão entre as vinte maiores causas de incapacidade em todo o mundo (Organização Mundial de Saúde, 2008). Aproximadamente metade da população mundial vive em países que, em média, possuem um psiquiatra para cada 200.000 pessoas. Em todo o mundo, o gasto com saúde mental é inferior a dois dólares anuais por pessoa, e menos de 25 centavos de dólar em países em desenvolvimento (Organização Mundial de Saúde, 2011a).

Provavelmente, nunca formaremos profissionais de saúde em número suficiente para administrar o cuidado adequado à saúde caso continuemos a confiar em intervenções que não podem ser usadas mais de uma vez. Por exemplo, uma sessão de terapia ou uma dose de medicamento só pode ser utilizada uma vez e o tempo usado no tratamento de um paciente não pode ser mais reutilizado para tratar outro paciente. Precisamos de intervenções que possam ser usadas mais de uma vez sem perder seu poder terapêutico e atingir pessoas que vivam em locais em que o sistema de saúde não consiga alcançar adequadamente, e que possam também ser distribuídas amplamente para a população (Muñoz, 2010).

Intervenções

comportamentais computadorizadas se enquadram nos critérios especificados acima, podendo ainda reduzir o impacto de outras limitações comuns nos tratamentos tradicionais, como baixas taxas de procura, baixas taxas de adesão e de evasão, recaídas e a estigmatização (Muñoz, 2010). Intervenções assistidas por computador focam principalmente questões comportamentais, com o objetivo de mudar um comportamento e causar melhorias de sintomas (Webb, Joseph, Yardley, \& Michie, 2010).

As intervenções comportamentais computadorizadas podem variar de acordo com sua base teórica, com as técnicas utilizadas para mudança de comportamentos e com a forma de disponibilização As bases teóricas podem ser definidas como referências a uma teoria ou teorias para o desenvolvimento de uma intervenção. Elas podem informar sobre a identificação de constructos utilizados como, por exemplo, atitudes, autoeficácia e autoestima, e sobre mecanismos relacionados a mudança de comportamentos, como o comportamento vicário e a seleção de participantes com maior probabilidade de uso, como pessoas com atitudes negativas, por exemplo (Webb et al., 2010). Entre as bases teóricas usadas em intervenções em transtornos de ansiedade e humor podem-se citar a teoria da cognição social e a teoria comportamental (Andersson \& Cuijpers, 2009; Cuijpers et al., 2009).

Não obstante, reina uma confusão quanto às técnicas utilizadas nas intervenções, sendo uma difícil tarefa determiná-las, devido a problemas de definição e diversidade (Webb et al., 2010). Por exemplo, dois estudos podem utilizar a mesma técnica para mudanças de comportamento e usar duas nomenclaturas diferentes para defini-las. A forma de disponibilização pode variar de acordo com os dispositivos utilizados - como computadores pessoais, telefones, tablets, palm tops e aparelhos de realidade virtual.

Com esta grande heterogeneidade, essas intervenções podem ser utilizadas: (1) quando nenhum outro tipo de tratamento está disponível; (2) quando os pacientes estão em listas de espera aguardando o tratamento; (3) como um adjunto ao tratamento padrão; (4) após o tratamento tradicional com o objetivo de prevenir recaídas; (5) para pacientes que não podem frequentar os centros de tratamento, devido à distância e limitações físicas ou econômicas; (6) para pacientes que se sentem estigmatizados ao procurar um centro de tratamento; e por último, (7) para estender o cuidado do tratamento à prevenção (Muñoz, 2010).

Existem evidências de eficácia destas intervenções para vários tipos de comportamentos e condições de saúde como o uso de álcool e outras drogas (Khadjesari, Murray, Hewitt, Hartley, \& Godfrey, 2011; Rooke, Thorsteinsson, Karpin, Copeland, \& Allsop, 2010), ansiedade (Cuijpers et al., 2009), depressão (Titov, Andrews, Schwencke, et al., 2010), transtornos alimentares (Schmidt et al., 2008), estresse (Ruwaard, Lange, Bouwman, Broeksteeg, \& Schrieken, 2007) e dor (Velleman, Stallard, \& Richardson, 2010).

Embora existam evidências de eficácia, as intervenções comportamentais computadorizadas ainda carecem de evidências de efetividade (Andersson, Carlbring, \& Cuijpers, 2009). Além disso, ainda se debate se 0 desenvolvimento destas intervenções tem acontecido por mero modismo, devido ao crescimento do uso de computadores e da internet nos países desenvolvidos e ao desenvolvimento de tecnologias (Marks, Cavanagh, \& Gega, 2007). O objetivo do presente estudo é analisar criticamente 
a literatura existente sobre intervenções comportamentais computadorizadas, a viabilidade dessas intervenções e o potencial de sua utilização no país.

\section{MÉTODO}

O presente estudo é uma revisão narrativa da literatura que busca levantar de forma não sistemática as principais referências bibliográficas sobre o tema proposto. A escolha deste método de revisão, em preferência a uma revisão sistemática da literatura, deveu-se à inexistência de estudos anteriores que abordassem a questão de pesquisa apresentada. Para tanto, inicialmente foram escolhidos como base para busca de material bibliográfico a Pubmed, que é a principal base de indexação de trabalhos científicos na área da saúde, e o periódico Journal of Medical Internet Research, que possui o maior fator de impacto na áreas relacionadas a intervenções comportamentais computadorizadas. A partir dos artigos encontrados, foram coletados artigos citados por estes que faziam referência ao tema tratado ou que subsidiavam a argumentação realizada neste trabalho.

\section{INTERVENÇÕES COMPORTAMENTAIS COMPUTADORIZADAS FUNCIONAM?}

Decerto, é praticamente impossível responder à questão com uma simples negativa ou afirmativa. A dificuldade encontrada é a mesma ao tentarmos responder a questão "Psicoterapia funciona?". Assim como as abordagens psicoterápicas, existem numerosos tipos de intervenções comportamentais e cada uma compreende um amplo conjunto de técnicas. Mesmo um tipo específico de abordagem - por exemplo, a cognitiva comportamental -, pode apresentar diferentes níveis de eficácia, uma vez que pode ser utilizada em diferentes modalidades (individual ou em grupos) e intervalos de tempo, e para diferentes tipos de transtorno e diferentes populações.

Assim, a pergunta adequada seria: "A técnica 'A da abordagem $B$ aplicada na modalidade $C$ para a população com transtorno D' funciona?". Para tentar responder de forma satisfatória à questão referente à eficácia das intervenções comportamentais computadorizadas serão apresentados estudos que apontam evidências de eficácia para diversas condições de saúde e os problemas comuns enfrentados pelos pesquisadores que tentam responder a esta questão. Por fim, serão apresentados alguns desafios que precisam ser contornados para que as evidências de eficácia sejam mais robustas.

\section{TABAGISMO}

O uso de tabaco é responsável por cinco milhões de mortes a cada ano, número maior que a soma das mortes por tuberculose, HIV/AIDS e malária (Organização Mundial de Saúde, 2009). Diversos ensaios clínicos randomizados e metaanálises foram realizados buscando avaliar a eficácia das intervenções comportamentais computadorizadas para tabagismo (Rooke et al., 2010). Uma pesquisa de meta-análise para avaliar a eficácia das intervenções comportamentais assistidas por computador para tabagismo revelou que essas apresentaram efeitos de pequenas dimensões (risco relativo $=1,44$ ) quando comparadas a grupos controles (Myung, McDonnell, Kazinets, Seo, \& Moskowitz, 2009). No mesmo estudo, ao serem realizadas análises de subgrupos, notou-se que efeitos significativos foram encontrados quando comparados os estudos com alta e baixa qualidade metodológica, indicando que os efeitos encontrados não estão relacionados a problemas de metodologia dos estudos.

\section{REDUÇÃO DO CONSUMO DE ÁLCOOL}

O excesso de uso de álcool é um dos maiores fatores de risco à saúde. É fator causal para mais de 60 tipos de doenças e acidentes, resultando em aproximadamente 2,5 milhões de mortes a cada ano (Organização Mundial de Saúde, 2011b). Grande parte das intervenções comportamentais computadorizadas busca a redução do consumo de álcool, e não o tratamento da dependência em si. Em um estudo de meta-análise foi verificado que as intervenções são mais eficazes que a simples avaliação do consumo através de um meio eletrônico; no entanto, deve-se observar que boa parte dos estudos clínicos randomizados que objetivam a redução do consumo de álcool inseridos em estudos de meta-análise apresentam problemas metodológicos que comprometem a validade da eficácia das intervenções como um todo. Estes problemas se devem a uma inapropriada escolha da medida de tendência central e os tamanhos das amostras (Khadjesari et 
al., 2011). Em outra meta-análise verificou-se que as intervenções para álcool apresentam efeitos maiores $(d=0,22)$ quando comparados o grupo intervenção e o de controle (Rooke et al., 2010).

\section{TRANSTORNOS DE ANSIEDADE}

Transtornos de ansiedade afetam uma a cada seis pessoas durante o curso de suas vidas (Somers, Goldner, Waraich, \& Hsu, 2006); no entanto, boa parte das pessoas que sofrem desses transtornos não buscam ajuda de um profissional de saúde. As intervenções comportamentais computadorizadas destinadas ao tratamento da ansiedade se fundamentam nas técnicas da terapia cognitiva comportamental (Titov, Andrews, Schwencke et al., 2010). Existem vários estudos clínicos randomizados que buscam avaliar a eficácia dessas psicoterapias para diferentes tipos de transtornos, como fobias (Titov, Andrews, Schwencke, et al., 2010), transtorno do estresse pós-traumático e transtorno do pânico (Cuijpers et al., 2009). Dois estudos de meta-análise foram conduzidos com o objetivo de avaliar a eficácia das intervenções para ansiedade (Cuijpers et al., 2009). Embora ambos tenham apresentado tamanhos de efeito significativos $(d=0,95$ e $d=0,75$, respectivamente) quando comparados os grupo intervenção e controle, ainda há limitações dos estudos quanto à quantidade dos ensaios clínicos randomizados analisados, aos níveis de abandono dos participantes e à falta de qualidade metodológica dos estudos. Apesar das limitações, os autores concluíram, preliminarmente, que os benefícios das intervenções computadorizadas são superiores aos da lista de espera e tratamento placebo (ex.: exercícios de relaxamento), e não são inferiores aos das intervenções terapêuticas tradicionais (Cuijpers et al., 2009).

\section{DEPRESSÃO}

A depressão é a terceira maior causa dos anos vividos com incapacidade e do tempo perdido devido à mortalidade prematura (Disability Adjusted Life Years ou DALY's) (Organização Mundial de Saúde, 2008). Existem diversos estudos que avaliaram a eficácia de intervenções comportamentais computadorizadas para depressão (Andersson \& Cuijpers, 2009; Kaltenthaler et al., 2008). Um estudo de metaanálise com quinze intervenções apresentou efeito moderado ( $d=0,37$ ), quando comparados os grupos intervenção e lista de espera (Andersson \& Cuijpers, 2009), tamanho de efeito comparável inclusive ao da terapia cognitiva comportamental tradicional ( $d=0,39$ ), quando comparados os grupos intervenção e lista de espera (Cuijpers, Smit, Bohlmeijer, Hollon, \& Andersson, 2010). Pela heterogeneidade das amostras e os diferentes delineamentos entre os estudos, pode-se inferir que intervenções com maiores níveis de suporte tendem a apresentar melhores tamanhos de efeito. Esse suporte é definido como qualquer contato realizado, independentemente de ter sido feito por um profissional de saúde ou por automação (Andersson \& Cuijpers, 2009). Em uma revisão de literatura sobre intervenções computadorizadas para depressão que utilizam técnicas da terapia cognitiva comportamental, foram encontradas altas taxas de abandono, variando entre $0 \%$ até $75 \%$, enquanto a porcentagem média do abandono $(31,75 \%)$ é DP=16,52) (Kaltenthaler et al., 2008).

\section{Transtornos alimentares, compulsão alimentar periódica e obesidade}

Duas pesquisas foram conduzidas para avaliar intervenções comportamentais computadorizadas para bulimia nervosa (Schmidt et al., 2008). As intervenções avaliadas tinham como objetivo ser um adjunto ao tratamento tradicional, e desta forma, reduzir o número de consultas durante o tratamento. As intervenções assistidas por computador para compulsão alimentar periódica têm como objetivo prevenir o aparecimento de transtornos alimentares mais graves e a obesidade. Os estudos apresentam evidências preliminares de eficácia para tais intervenções (Robinson \& Serfaty, 2008). Um estudo avaliou uma intervenção desenvolvida para ser utilizada como ferramenta para educação em relacionamento e melhoria de indicadores de saúde mental como depressão e ansiedade. Os resultados apontaram que 0 programa funcionou melhor que o grupo controle, tanto para melhorar as habilidades envolvidas em relacionamentos quanto para melhorar a saúde mental geral dos participantes do grupo intervenção (Braithwaite \& Fincham, 2009).

\section{DOR}

Apenas um estudo de meta-análise analisou a eficácia de intervenções baseadas na terapia cognitiva comportamental para redução da dor em 
crianças e adolescentes. Nesse estudo, apenas quatro ensaios clínicos foram avaliados. O tamanho de efeito encontrado foi moderado ( $d=-0,41$ efeito negativo, visto que escores menores indicam menores sinais de dor), considerado moderado quando comparados os grupos intervenção e a lista de espera (Velleman, Stallard, \& Richardson, 2010); porém o estudo deve ser observado com cautela, visto que as amostras incluídas não apresentaram validade externa e os grupos de comparação utilizados eram de listas de espera, por isso deverão ser realizadas pesquisas com desenhos metodológicos mais elegantes.

\section{Outras condições de saúde}

Existem estudos clínicos randomizados e não controlados para outros tipos de transtornos e condições de saúde, como dependência de substâncias (Carroll et al., 2008) e estresse ligado ao trabalho (Ruwaard et al., 2007), embora não existam meta-análises publicadas que avaliem a eficácia desses estudos. Em um estudo para avaliar a eficácia de uma intervenção por computador para dependentes de substâncias em que se utilizou como base a terapia cognitiva comportamental verificou-se que os participantes utilizaram menos substâncias do que os que participaram do tratamento tradicional $(d=0,59)$, quando comparados os grupo controle e lista de espera (Carroll et al., 2008). O programa de intervenção foi utilizado dentro de uma clínica, com suporte de um profissional.

Para reduzir o estresse no trabalho, foi desenvolvida uma intervenção baseada na terapia cognitiva comportamental (Ruwaard et al., 2007). $O$ programa foi criado para otimizar o tempo do atendimento psicoterapêutico, consistindo no envio de material de autoajuda e acompanhamento através de e-mails por clínicos treinados. A intervenção apresentou tamanho de efeito moderado para redução do estresse no trabalho (d $=0,60$ ), quando comparados os grupo intervenção e lista de espera. O tamanho de efeito foi consistente $(d=0,70)$ quando comparados ao de um estudo que utilizou uma intervenção por internet (Zetterqvist, Maanmies, Ström, \& Andersson, 2003).

\section{Desafios em pesquisas com intervenções comportamentais computadorizadas}

Diversos desafios permeiam a realização de estudos para avaliar a eficácia das intervenções comportamentais computadorizadas. O primeiro desafio é a falta de um protocolo adequado para o desenvolvimento de desenhos e ensaios clínicos para essa modalidade de intervenção. O grupo CONSORT (Consolidated Standards of Reporting Trials), que tem como missão melhorar a qualidade dos ensaios clínicos randomizados, testa um checklist para avaliação de estudos sobre essas intervenções (Consolidated Standards of Reporting Trials, 2011).

O segundo desafio a ser superado são as taxas de perda de participantes durante a realização dos ensaios clínicos. Essas taxas podem comprometer gravemente a validade externa dos estudos. O terceiro desafio a ser superado é a heterogeneidade das intervenções. Falta definição conceitual das técnicas e bases teóricas descritas nos estudos (Webb et al., 2010). Somados à falta de definição, existem problemas das medidas utilizadas nos estudos e análise dos dados, salvo os estudos com intervenções para tabagismo, que apresentam recomendações para as medidas de desfecho para análise dos dados e tratamento de dados faltosos.

\section{É hora de investirmos nas intervenções comportamentais computadorizadas no brasil?}

Apresentadas as evidências de eficácia das intervenções comportamentais computadorizadas para diversos tipos de comportamentos, é preciso analisar o contexto brasileiro, através do padrão de acesso às tecnologias e à legislação existente no país. Também são apresentadas ferramentas para o desenvolvimento e avaliação de tais intervenções.

\section{Aumento do acesso a tecnologias no brasil: computadores, internet e celulares}

Entre os anos de 2009 e 2010 a proporção de domicílios brasileiros com um computador cresceu de $32 \%$ para $35 \%$. Nos últimos seis anos a proporção de domicílios com computador mais que dobrou na área urbana; Mas mesmo com esse crescimento, ainda existe um alto nível de exclusão digital no país, principalmente na área rural (Comitê Gestor da Internet no Brasil, 2011).

A internet se configura como um importante meio de acesso à informação e um potencial mecanismo de disponibilização de intervenções computadorizadas, sendo utilizada por aproximadamente dois bilhões de pessoas em todo o mundo. Na última década o acesso à Internet vem crescendo em todo o mundo (584,4\%), principalmente na América Latina (1.039,0\%). O Brasil representa 46,7\% dos acessos na América 
Latina, totalizando aproximadamente 76 milhões de usuários, o que equivale a $37,4 \%$ de toda a população (Internet World Stats, 2012). De forma geral, nos últimos anos ocorreu no Brasil aumento do uso tanto de computadores quanto da internet. $\mathrm{Na}$ área urbana, por exemplo, o uso diário da Internet subiu de 40\% em 2005 para 60\% em 2010, um crescimento de $50 \%$ no período (Comitê Gestor da Internet no Brasil, 2011). Entre as atividades realizadas na internet destacam-se o envio e recebimento de e-mails (80\%), o envio e recebimento de mensagens instantâneas $(74 \%)$ e a participação em sites de relacionamentos (70\%).

Em relação à busca de informações realizadas, destacam-se pesquisas sobre diversão e entretenimento (61\%), bens e serviços $(58 \%)$ e saúde e serviços de saúde (35\%). Entre as dificuldades mais recorrentes do uso da internet destacam-se: acessar sites ou páginas que demoram muito, muito pesadas (46\%); não encontrar a informação desejada no site (35\%); ler um texto longo (29\%); e acessar páginas com janelas que aparecem na tela (28\%). Os problemas investigados podem ser decorrentes de páginas confusas e de difícil navegação, o que muitas vezes está relacionado ao desenvolvimento dos websites e à arquitetura das páginas (Comitê Gestor da Internet no Brasil, 2011).

Outro meio potencial para o desenvolvimento de intervenções comportamentais computadorizadas no Brasil é a telefonia celular. Em 2010, 79\% dos brasileiros afirmaram ter usado um aparelho celular pelo menos uma vez nos últimos três meses. A posse de aparelhos apresenta crescimento desde 2005, principalmente nas áreas rurais e menos desenvolvidas economicamente e entre as pessoas pertencentes às classes $D$ e $E$ e as de baixa escolaridade (Comitê Gestor da Internet no Brasil, 2011).

O potencial da internet para a realização de pesquisas e intervenções comportamentais no país pode ser observado no estudo Brazilian Internet Study on Temperament and Psychopathology (BRAINSTEP) na área de psiquiatria e psicologia. O estudo é realizado através de um site (http://www.temperamento.com.br) com questionários e instrumentos disponibilizados gratuitamente, com o objetivo de avaliar o temperamento e psicopatologias dos entrevistados. Através do website deste estudo foram recolhidos, entre dezembro de 2009 e abril de 2010, dados validados de aproximadamente 30.000 sujeitos (Lara, Ottoni, Brunstein, Frozi, de Carvalho \& Bisol, 2012).

\section{REGULAMENTAÇÕES EXISTENTES}

No Brasil não existem leis específicas que regulamentem as intervenções comportamentais por internet, embora existam regulamentações dos conselhos federais de medicina e psicologia que as tangem. A Resolução $n^{\circ}$ 1.639/2002 do Conselho de Ética Médica define as normas técnicas para o uso de sistemas informatizados para a guarda e manuseio do prontuário médico. Ela dispõe sobre o tempo de guarda dos prontuários e estabelece os critérios para certificação dos sistemas de informação. Segundo a referida resolução, o sistema de informação deve ser capaz de identificar usuários através de algum método de autenticação, sendo necessária a coleta de registros de eventos que contenham os seguintes dados: identificação do usuário; datas e horários de entrada e saída; identidade do terminal de acesso e, quando possível, sua localização; registro das tentativas aceitas e rejeitadas de acesso ao sistema; e registro das tentativas de acesso ao sistema. A Resolução também determina que, para ser realizada a transmissão de prontuários, os sistemas deverão apresentar certificação digital emitida por um órgão competente (Conselho Federal de Medicina, 2002).

Existem cinco resoluções do Conselho Federal de Psicologia relacionadas ao atendimento à distância. A Resolução $\mathrm{n}^{\circ} \quad 002 / 95$ veda ao psicólogo quaisquer tipos de prestação de serviços, ou mesmo a vinculação do título de psicólogo a serviços de atendimento psicológico via telefone. A Resolução $n^{\circ}$ 01/2012 revoga a Resolução $n^{\circ}$ 012/2005 e amplia a utilização da utilização de meios tecnológicos de comunicação à distância. Essa resolução permite os serviços psicológicos desde que sejam pontuais e educativos, focalizem o tema proposto e não firam o código de ética da profissão. Fica permitida a realização de orientações psicológicas, sendo estas definidas como orientação ou atendimento realizado em até vinte encontros ou contato virtuais, síncronos ou assíncronos, processos prévios de seleção de pessoas, aplicação de testes, supervisão de trabalho de psicólogos e atendimento virtual de clientes em trânsito ou que se apresentem momentaneamente impossibilitados de comparecer ao atendimento presencial.

A referida resolução possibilita a utilização de softwares informativos e educativos com resposta automatizada, desde que sejam garantidas as seguintes condições: (1) os psicólogos 
responsáveis serem identificados através de credencial - na forma de selo ou equivalente -, desenvolvida e conferida pelo Conselho Federal de Psicologia; (2) o psicólogo responsável técnico estar regularmente inscrito no Conselho Regional de Psicologia e em pleno gozo de seus direitos; (3) o psicólogo responsável dirigir requerimento informando as características dos serviços disponibilizados; (4) o psicólogo fazer 0 cadastramento anual do serviço disponibilizado pelo site. Para fins não psicoterapêuticos, é permitida a cobrança de honorários, desde que se respeite o Código de Ética da Profissão. Para validar, acompanhar e fiscalizar os serviços de psicologia pela internet, o Conselho Federal de Psicologia instituiu a Comissão Nacional de Credenciamento de sites através da Resolução $n^{\circ}$ 006/2000 (Conselho Federal de Psicologia, 2012).

Ferramentas para o desenvolvimento das intervenções comportamentais computadorizadas

Para realização de intervenções comportamentais computadorizadas é recomendável a articulação entre profissionais de saúde e profissionais da tecnologia da informação; no entanto, com o advento dos sistemas de gerenciamento de conteúdo Wordpress e Drupal, o desenvolvimento e manutenção destas intervenções podem ser realizados com custos razoavelmente baixos. Um desses sistemas utilizados para desenvolvimento de intervenções comportamentais computadorizadas é o LifeGuide (http://www.lifeguideonline.org).

O sistema LifeGuide é mantido pelo Conselho de Pesquisa Econômica e Social do Reino Unido, constituindo parte do programa de Pesquisa Social Digital. Embora situado no Reino Unido, é permitido a qualquer profissional 0 desenvolvimento de intervenções e posterior avaliação de sua eficácia. A partir de uma lógica de programação simplificada, o sistema permite: (1) o desenvolvimento e cotação de questionários, (2) feedback personalizado a partir de respostas fornecidas pelo usuário, (3) automação de envio de e-mails e mensagens de textos, (4) o planejamento, a análise gráfica e consultas a seu progresso por parte dos usuários, (5) a randomização de participantes; e (6) o arquivamento de todos os dados relativos às respostas dos usuários e aos registros de utilização do site. Os servidores ficam hospedados na Southampton University e todos os dados são guardados com a utilização de uma política de segurança conforme com os padrões internacionais. Para facilitar a utilização do sistema são disponibilizados manuais, exemplos e workshops. Caso seja necessário, após o desenvolvimento, pode ser realizada a migração da intervenção para um servidor próprio.

\section{CONSIDERAÇÕES FINAIS}

O Brasil possui dimensões continentais e o acesso às intervenções tradicionais pelo sistema público ainda é muito limitado. As intervenções comportamentais podem constituir um importante complemento para melhorar a saúde, uma vez que apresentam alcance à crescente proporção da população com acesso a computadores e à internet. As regulamentações atuais do Conselho Federal de Medicina e do Conselho Federal de Psicologia possibilitam um contexto para 0 desenvolvimento e pesquisas de avaliação. Mesmo com os orçamentos limitados oferecidos pelas agências de fomento à pesquisa no país, a avaliação da eficácia clínica destas intervenções é possibilitada pelo baixo custo de ferramentas e plataformas de avaliação como, por exemplo, o Lifeguide. Os custos de uma pesquisa de avaliação clínica de uma intervenção computadorizada podem ser considerados menores quando comparados ao custo da avaliação de uma intervenção presencial, em que é necessário gastar recursos com o treinamento e pagamento de profissionais de saúde, impressão, coleta e digitação dos questionários de pesquisa nas fases de intervenção e nos meses de seguimento. Considerando-se os argumentos apresentados no presente trabalho, estamos numa boa hora de investir no desenvolvimento e avaliação destas intervenções.

\section{REFERÊNCIAS}

Andersson, G., Carlbring, P., \& Cuijpers, P. (2009). Internet interventions: Moving from efficacy to effectiveness. E-Journal of Applied Psychology, 5(2), 18-24.

Andersson, G., \& Cuijpers, P. (2009). Internet-based and other computerized psychological treatments for adult depression: A meta-analysis. Cognitive Behaviour Therapy, 38(4), 196-205. doi:10.1080/16506070903318960

Braithwaite, S. R., \& Fincham, F. D. (2009). A randomized clinical trial of a computer based preventive intervention: Replication and 
extension of ePREP. Journal of Family Psychology, 23(1), 32-38. doi:10.1037/a0014061

Carroll, K. M., Ball, S. A., Martino, S., Nich, C., Babuscio, T. A., Nuro, K. F., Gordon, M. A., et al. (2008). Computer-assisted delivery of cognitivebehavioral therapy for addiction: A randomized trial of CBT4CBT. The American Journal of Psychiatry, 165(7), doi:10.1176/appi.ajp.2008.07111835

Comitê Gestor da Internet no Brasil. (2011). Pesquisa sobre o uso das tecnologias de informação e comunicação no Brasil:TIC Domicílios e TIC Empresas (Vol. 1). Recuperado em 10/05/2011 de http://www.cetic.br

Conselho Federal de Medicina. (2002). Resolução CFM no $1.643 / 2002$ Define e disciplina a prestação de serviços através da Telemedicina. Recuperado em10/05/2011 de: http://www.portalmedico.org.br/resolucoes/cfm/20 02/1643 2002.htm

Conselho Federal de Psicologia. (2012). Resolução CFP $n^{O}$ 011/2012 - Regulamenta o atendimento psicoterapêutico e outros serviços psicológicos mediados por computador e revoga a Resolução CFP $N^{\circ}$ 012/2005. Recuperado em 01/08/2012 de:

http://www.crpsp.org.br/portal/orientacao/resoluc oes_cfp/fr_cfp_011-12.aspx

Consolidated Standards of Reporting Trials. (2011). Consort E-health checklist (V.1.6.1): Information to include when reporting ehealth/mhealth trials. Recuperado em 10/05/2012 de: http://www.jmir.org/ojs/public/journals/1/CONSOR T-EHEALTH-v1-6.pdf

Cuijpers, P., Marks, I. M., van Straten, A., Cavanagh, K., Gega, L., \& Andersson, G. (2009). Computer-aided psychotherapy for anxiety disorders: A meta-analytic review. Cognitive Behaviour Therapy, 38(2), 66-82. doi:10.1080/16506070802694776

Cuijpers, P., Smit, F., Bohlmeijer, E., Hollon, S. D., \& Andersson, G. (2010). Efficacy of cognitivebehavioural therapy and other psychological treatments for adult depression: meta-analytic study of publication bias. The British Journal of Psychiatry, 196, doi:10.1192/bjp.bp.109.066001

Internet World Stats. (2012). Internet World Stats. Recuperado em 10/05/2011 de: http://www.internetworldstats.com

Kaltenthaler, E., Sutcliffe, P., Parry, G., Beverley, C., Rees, A., \& Ferriter, M. (2008). The acceptability to patients of computerized cognitive behaviour therapy for depression: A systematic review. Psychological Medicine: A Journal of Research in Psychiatry and the Allied Sciences, 38(11), 15211530. doi:10.1017/S0033291707002607

Khadjesari, Z., Murray, E., Hewitt, C., Hartley, S., \& Godfrey, C. (2011). Can stand-alone computerbased interventions reduce alcohol consumption? A systematic review. Addiction, 106(2), 267-282. doi:10.1111/j.1360-0443.2010.03214.x
Lara, D. R., Ottoni, G. L., Brunstein, M. G., Frozi, J., de Carvalho, H. W., \& Bisol, L. W. (2012). Development and validity data of the Brazilian Internet Study on Temperament and Psychopathology (BRAINSTEP). Journal of Affective doi:10.1016/j.jad.2012.03.011

Marks, Cavanagh, K., \& Gega, L. (2007). Computeraided psychotherapy: Revolution or bubble? British Journal of Psychiatry, 191(6), 471-473. doi:10.1192/bjp.bp.107.041152

Muñoz, R. F. (2010). Using Evidence-Based Internet Interventions to Reduce Health Disparities Worldwide. Journal of Medical Internet Research, 12. doi:10.2196/jmir.1463

Myung, S.K., McDonnell, D. D., Kazinets, G., Seo, H. G., \& Moskowitz, J. M. (2009). Effects of Weband Computer-Based Smoking Cessation Programs: Meta-analysis of Randomized Controlled Trials. Archives of Internal Medicine, 169(10), 929-937.

Organização Mundial de Saúde. (2008). The global burden of disease 2004 update. Genebra: Organização Mundial de Saúde.

Organização Mundial de Saúde. (2009). Global health risks: mortality and burden of disease attributable to selected major risks. Genebra: Organização Mundial de Saúde.

Organização Mundial de Saúde. (2011a). Mental health atlas 2011. Genebra: Organização Mundial de Saúde.

Organização Mundial de Saúde. (2011b). Global status report on alcohol and health. Genebra: Organização Mundial de Saúde.

Robinson, P., \& Serfaty, M. (2008). Getting better byte by byte: A pilot randomised controlled trial of email therapy for bulimia nervosa and binge eating disorder. European Eating Disorders Review, 16(2), 84-93. doi:10.1002/erv.818

Rooke, S., Thorsteinsson, E., Karpin, A., Copeland, J., \& Allsop, D. (2010). Computer-delivered interventions for alcohol and tobacco use: A meta-analysis. Addiction, 105(8), 1381-1390. doi:10.1111/j.1360-0443.2010.02975.x

Ruwaard, J., Lange, A., Bouwman, M., Broeksteeg, J., \& Schrieken, B. (2007). E-mailed standardized cognitive behavioural treatment of work-related stress: A randomized controlled trial. Cognitive Behaviour Therapy, 36(3), 179-192. doi:10.1080/16506070701381863

Schmidt, U., Andiappan, M., Grover, M., Robinson, S., Perkins, S., Dugmore, O., Landau, S., et al. (2008). Randomised controlled trial of CD-ROMbased cognitive-behavioural self-care for bulimia nervosa. British Journal of Psychiatry, 193(6), 493-500. doi:10.1192/bjp.bp.107.046607

Somers, J. M., Goldner, E. M., Waraich, P., \& Hsu, L. (2006). Prevalence and incidence studies of anxiety disorders: a systematic review of the literature. Canadian Journal of Psychiatry. Revue Canadienne De Psychiatrie, 51(2), 100-113. 
Titov, N., Andrews, G., Schwencke, G., Robinson, E., Peters, L., \& Spence, J. (2010). Randomized controlled trial of internet cognitive behavioural treatment for social phobia with and without motivational enhancement strategies. Australian and New Zealand Journal of Psychiatry, 44(10), 938-945. doi:10.3109/00048674.2010.493859

Velleman, S., Stallard, P., \& Richardson, T. (2010). $A$ review and meta-analysis of computerized cognitive behaviour therapy for the treatment of pain in children and adolescents. Child: care, health and development, 36(4), 465-472. doi:10.1111/j.1365-2214.2010.01088.x

ebb, T. L., Joseph, J., Yardley, L., \& Michie, S. (2010). Using the Internet to Promote Health Behavior Change: A Systematic Review and Meta-analysis of the Impact of Theoretical Basis, Use of Behavior Change Techniques, and Mode of Delivery on Efficacy. Journal of Medical Internet Research, 12. doi:10.2196/jmir.1376
Zetterqvist, K., Maanmies, J., Ström, L., \& Andersson, G. (2003). Randomized controlled trial of internet-based stress management. Cognitive Behaviour Therapy, 32(3), 151-160. doi:10.1080/16506070302316.

Henrique Pinto Gomide: mestrando em Psicologia pelo Programa de Pós-Graduação em Psicologia da Universidade Federal de Juiz de Fora, pesquisador do Centro de Pesquisa, Intervenção e Avaliação em Álcool e outras Drogas.

Leonardo Fernandes Martins: Mestre em Psicologia pela Universidade Federal de Juiz de Fora, onde contou com o financiamento do CNPq, professor do curso de Psicologia da Fundação Presidente Antônio Carlos.

Telmo Mota Ronzani: doutor em Ciências da Saúde pela Universidade Federal de São Paulo, pós-doutorado pela Universidade de São Paulo e estágio pós-doutoral pela University of Connecticut Health Center, professor adjunto da Universidade Federal de Juiz de Fora e professor do curso de pós-graduação strito senso em Saúde Brasileira e Psicologia da UFJF. Bolsista de Produtividade (CNPq). 